\title{
STATISTICAL PARAMETERS TO ESTIMATE THE LEAF AREA OF NATIVE FOREST SEEDLINGS OF GENUS Tabebuia AND Handroanthus
}

\author{
PARÂMETROS ESTATÍSTICOS PARA ESTIMAR A ÁREA FOLIAR DE MUDAS DE \\ ESPÉCIES NATIVAS DOS GENNEROS Tabebuia $e$ Handroanthus
}

\begin{abstract}
Emanoeli Borges MONTEIRO'; Andréa Carvalho da SILVA²; Adilson Pacheco de SOUZA²; Cátia Cardoso da SILVA ${ }^{3}$; Verônica Satomi KAZAMA ; Adriana Aki TANAKA ${ }^{5}$

1. Mestre em Agronomia, Instituto de Ciências Agrárias e Ambientais, Universidade Federal de Mato Grosso - UFMT, Campus Sinop, MT, Brasil. emanoeliborges@yahoo.com.br; 2. Professor (a), Doutor (a), Instituto de Ciências Agrárias e Ambientais, Universidade Federal de Mato Grosso - UFMT, Campus Sinop, MT, Brasil; 3. Mestranda em Ciências Ambientais, Instituto de Ciências Naturais, Humanas e Sociais, Universidade Federal de Mato Grosso - UFMT, Campus Sinop, MT, Brasil; 4. Mestranda em Engenharia Florestal, Centro de Ciências Rurais, Universidade Federal de Santa Maria, Campus Santa Maria, RS, Brasil; 5. Pesquisadora Associada, Doutora em Agronomia, Instituto de Ciências Agrárias e Ambientais, Universidade Federal de Mato Grosso - UFMT, Campus Sinop, MT, Brasil.
\end{abstract}

\begin{abstract}
Leaf area (LA) is an important parameter for physiological and phytotechnical studies and its measurement in a fast, accurate, and inexpensive way is essential and desirable. In this context, mathematical modeling is used as a tool to estimate leaf area from its relation with biometrical parameters and biomass. This study aimed to generate, validate, and determine the best mathematical estimation models of leaf area using the linear variables length (with and without petiole) and width of leaves and leaflets, in addition to dry mass of the native species Tabebuia roseoalba, Tabebuia impetiginosa and Handroanthus chrysotrichus collected in Sinop, Mato Grosso State (Brazil), between January and March 2014. The model assessment was performed by the method of weighted values of statistical indicators. The models based on linear measurements as independent variable that provided best performance of LA estimation for T. impetiginosa and T. roseoalba use the average leaflet width (Wla) measurements: LA=10.919 $\times$ Wla. ${ }^{1.854}$ and $\mathrm{LA}=6.196 \times \mathrm{Wla}^{1.684}$, respectively. For $H$. chrysotrichus, the model was based on the length and width of leaves ( $\mathrm{L}$ and $\mathrm{W}): \mathrm{LA}=(0.383 \times \mathrm{L} \times \mathrm{W})+16.586$. The best models of leaf area estimation considering dry mass (DM) were $\mathrm{LA}=119.510 \times \mathrm{DM}-32.044 \times \mathrm{DM}^{2}$ for $H$. chrysotrichus, $\mathrm{LA}=143.610 \times \mathrm{DM}-6.383 \times \mathrm{DM}^{2}$ for $T$. impetiginosa, and $\mathrm{LA}=90.623 \times \mathrm{DM}$ for $T$. roseoalba.
\end{abstract}

KEYWORDS: Biometrics. Foliar measurements. Regression analysis. Statistical indicator.

\section{INTRODUCTION}

Leaf area (LA) is an important physiological parameter for studying growth, development, and productivity of plant species considering its relation with processes such as photosynthesis, transpiration, use of light, water and nutrients, radiation interception and energy balance (SMART, 1985; WILLIAMS, 1987; GARDNER et al., 1990; FAVARIN et al., 2002). Its acquisition allows assessing the specific leaf area, net-radiation absorption, evapotranspiration intensity, leaf area ratio, leaf area index, canopy aerodynamic resistance, soil shading, among other interactions with the environment (SILVA et al., 2011; SOUZA et al., 2014).

LA is also an important biometric parameter for assessing plant responses to different environmental conditions. Easy, fast, and nondestructive methods that accurately estimate LA are important for assessing plant growth under field conditions, especially for perennial crops and forest species at initial development stages. According to Aquino et al. (2011), LA quantification can be performed by direct or indirect methods.

Destructive direct methods (as leaf area integrator and weighing) are usually more laborious and demanding time (TOEBE et al., 2012). In these methods the leaves are harvested in order to be evaluated, therefore, plant structure is damaged, becoming impossible carry out successive measurements in the same plant (GIUFFRIDA et al., 2011).

Non-destructive indirect methods present as characteristics a reduced time spent (SERDAR; DEMIRSOY, 2006), greater precision, as well as the possibility of monitoring leaf growth and leaf expansion throughout plant cycle or experiment, reducing data variability (MARSHALL, 1968; PEKSEN, 2007; TOEBE et al., 2012). These methods are indicated for in loco assessments and are useful for studies that require non-destructive methods such as photosynthesis and transpiration (NASCIMENTO et al., 2002).

Mathematical relations that measure LA using isolated or combined linear dimensions (length and width) of the leaf are usually the most reported in the literature (KVET; MARSHALL, 
1971; SMITH; KLIEWER, 1984; ELSNER; JUBB, 1988; MONTERO et al., 2000; SOUZA et al., 2014). Furthermore, petiole length can also be used (MANIVEL; WEAVER, 1974; MONTERO et al., 2000) as fresh or dry mass of leaves (SEPÚLVEDA; KLIEWER, 1983; MONTERO et al., 2000; CHO et al., 2007).

Considering the information provided by LA and the great biodiversity and potential presented by tropical forest species, the morphological knowledge about them is incipient and extremely necessary. Thus, this study aimed to generate, validate and determine the best estimation models of LA based on linear measurements and dry mass of leaves and leaflets for three tropical native species: Handroanthus chrysotrichus, Tabebuia impetiginosa, and Tabebuia roseoalba.

\section{MATERIAL AND METHODS}

The experiment was carried out at the Federal University of Mato Grosso, located in Sinop $\left(11^{\circ} 51^{\prime} 08^{\prime \prime} \mathrm{S}, 55^{\circ} 30^{\prime} 56^{\prime \prime} \mathrm{W}\right.$, and 376 meters above sea level), in the Cerrado-Amazon transition region, northern Mato Grosso, Brazil. Regional climate is classified as Aw according to Köppen, i.e. a tropical warm and humid climate, with two well-defined stations: a rainy season from October to April and a dry season from May to September. In addition, this region presents low annual thermal amplitude with a monthly average between 23.5 and $25.5{ }^{\circ} \mathrm{C}$ and an annual average precipitation of between 1327.29 and $1974.47 \mathrm{~mm}$ (SOUZA et al., 2013).

From January to March 2014, 250 leaves fully expanded, without deformations, non-damaged and with different sizes were collected from the forest species Handroanthus chrysotrichus (Mart. ex A. DC.) Mattos, Tabebuia impetiginosa (Mart. ex DC.) Standl., and Tabebuia roseoalba (Rid.) Sand. An average of five tree matrices with good phytosanitary conditions were used per species. The studied species have compound leaves (leaf blade divided into leaflets) (Figure 1) with three or more leaflets emerging from the apex of the main petiole (VIDAL; VIDAL, 2003).

The maximum length and width of leaves ( $\mathrm{L}$ and $\mathrm{W}$ ) and their leaflets ( $\mathrm{Ll}$ and $\mathrm{Wl}$ ) were measured by using a ruler and measuring tape $(\mathrm{cm})$. The generated analytical models considered the presence and absence of petiole in the leaves for $\mathrm{L}$. In the first case, $\mathrm{L}$ is the distance from the base of petiole to leaf apex; in the second case, $\mathrm{L}$ is the distance between the petiole insertion point in the leaf blade and the leaf apex. For leaflets, $\mathrm{Ll}$ is the distance between the leaflet apex and its insertion in the rachis whereas $\mathrm{W}$ and $\mathrm{Wl}$ are the larger perpendicular distance to the longitudinal axis ( $\mathrm{L}$ or Ll) of leaves and leaflets, respectively.

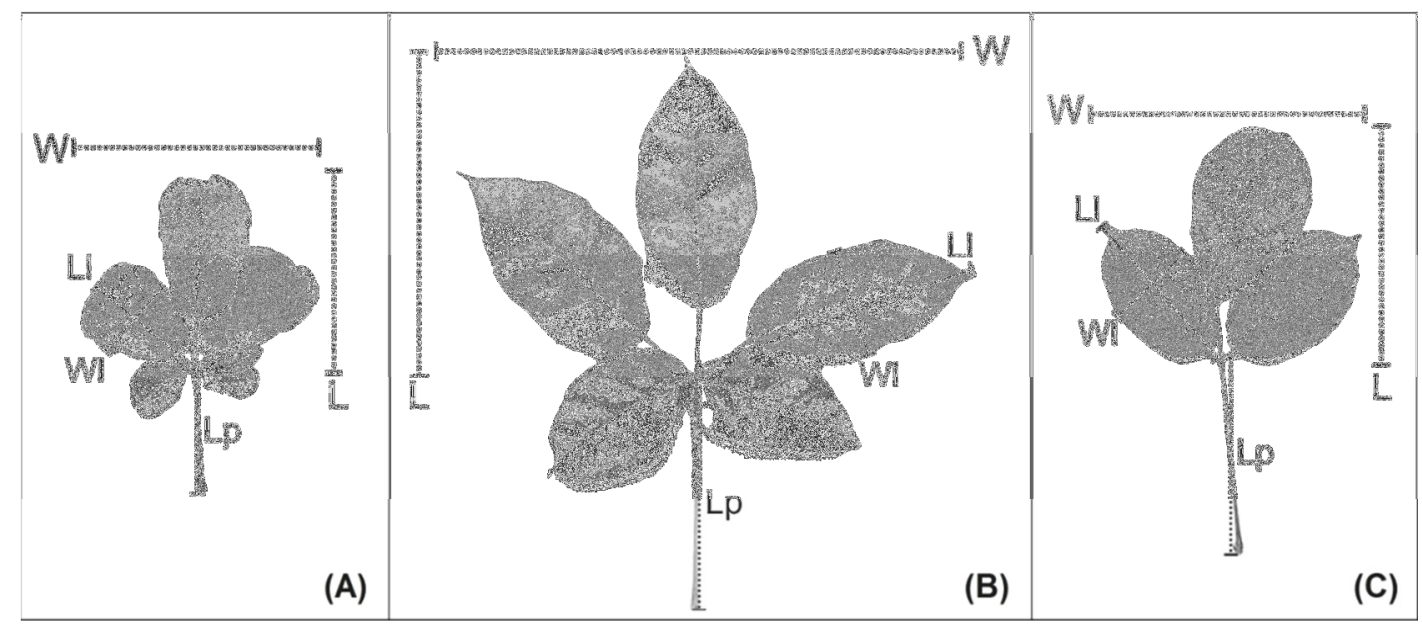

Figure 1. Leaves of studied species: (A) Handroanthus chrysotrichus, (B) Tabebuia impetiginosa and (C) Tabebuia roseoalba. Where: L - leaf length, $\mathrm{W}$ - leaf width, Lp - petiole length, $\mathrm{Ll}$ - leaflet length, and $\mathrm{Wl}$ - leaflet width.

After the linear parameters were measured, the real leaf area (LA, in $\mathrm{cm}^{2}$ ) was determined by using a photoelectric meter (Li-3000 Model, Li-Cor, Lincoln, NE, USA). Subsequently, dry mass of leaves (DM, in $\mathrm{g}$ ) was obtained by weighing the plant material in a precision balance $(0.001 \mathrm{~g})$ after being dried in a forced air circulation oven at $65 \pm 5$
${ }^{\circ} \mathrm{C}$ until constant weight. All collected leaves (250) were used for linear measurements and leaf area without petiole, whereas 50 leaves were used for measurements with petiole, in addition to dry mass measurements (with and without petiole).

With the obtained data, analytical models of leaf area estimation were generated and validated. 
For the model generation, leaf characteristics were considered aiming at establishing the highest number of simplified mathematical relations among the measurements performed. Therefore, based on the mathematical combinations proposed by Souza et al. (2014), 40 analytical models (Table 1) were adapted, 36 of them for linear measurements (24 considering the leaflets) and the others for dry mass.

Table 1. Analytical models generated for the species applying linear measurements or dry mass. Where: $\mathrm{L}-$ leaf length $(\mathrm{cm}) ; \mathrm{W}$ - leaf width $(\mathrm{cm}) ; \mathrm{Ll}_{\mathrm{n}}$ - leaflet length $\mathrm{n}(\mathrm{cm}) ; \mathrm{Wl}_{\mathrm{n}}$ - leaflet width $\mathrm{n}(\mathrm{cm}) ; \mathrm{n}-$ number of leaflets; Lla - average leaflet length $(\mathrm{cm})$; Wla - average leaflet width $(\mathrm{cm}) ; \mathrm{a}_{\mathrm{n}}$ and $\mathrm{b}-$ adjusted coefficients; and DM - dry mass (g).

\begin{tabular}{|c|c|c|}
\hline \multicolumn{3}{|c|}{ Analytical models based on linear measures } \\
\hline $\mathbf{1}$ & $\mathrm{LA}=\mathrm{a}_{1} \times \mathrm{L}$ & $19 \mathrm{LA}=\mathrm{a}_{1} \times\left(\mathrm{Lla}^{\mathrm{b}}\right)$ \\
\hline 2 & $\mathrm{LA}=\left(\mathrm{a}_{1} \times \mathrm{L}\right)+\mathrm{a}_{2}$ & $20 \mathrm{LA}=\left(\mathrm{a}_{1} \times \mathrm{Wl}_{1}\right)+\ldots+\left(\mathrm{a}_{\mathrm{n}} \times \mathrm{Wl} \mathrm{l}_{\mathrm{n}}\right)$ \\
\hline & $\mathrm{LA}=\mathrm{a}_{1} \times\left(\mathrm{L}^{\mathrm{b}}\right)$ & $21 \mathrm{LA}=\mathrm{a}_{1} \times\left(\mathrm{Wl}_{1}+\ldots+\mathrm{Wl} \mathrm{l}_{\mathrm{n}}\right)$ \\
\hline 4 & $\mathrm{LA}=\mathrm{a}_{1} \times \mathrm{W}$ & $22 \mathrm{LA}=\left[\mathrm{a}_{1} \times\left(\mathrm{Wl}_{1}+\ldots+\mathrm{Wl} \mathrm{l}_{\mathrm{n}}\right)\right]+\mathrm{a}_{2}$ \\
\hline 5 & $\mathrm{LA}=\left(\mathrm{a}_{1} \times \mathrm{W}\right)+\mathrm{a}_{2}$ & $23 \mathrm{LA}=\mathrm{a}_{1} \times\left[\left(\mathrm{Wl}_{1}+\ldots+\mathrm{Wl} \mathrm{l}_{\mathrm{n}}\right)^{\mathrm{b}}\right]$ \\
\hline & $\mathrm{LA}=\mathrm{a}_{1} \times\left(\mathrm{W}^{\mathrm{b}}\right)$ & $24 \mathrm{LA}=\mathrm{a}_{1} \times \mathrm{Wla}$ \\
\hline 7 & $\mathrm{LA}=\mathrm{a}_{1} \times \mathrm{L} \times \mathrm{W}$ & $25 \mathrm{LA}=\left(\mathrm{a}_{1} \times \mathrm{Wla}\right)+\mathrm{a}_{2}$ \\
\hline 8 & $\mathrm{LA}=\left(\mathrm{a}_{1} \times \mathrm{L} \times \mathrm{W}\right)+\mathrm{a}_{2}$ & $26 \mathrm{LA}=\mathrm{a}_{1} \times\left(\mathrm{Wla}^{\mathrm{b}}\right)$ \\
\hline 9 & $\mathrm{LA}=\mathrm{a}_{1} \times(\mathrm{L}+\mathrm{W})$ & $27 \mathrm{LA}=\mathrm{a}_{1} \times\left(\mathrm{Ll}_{1}+\mathrm{Wl}_{1}\right)+\ldots+\mathrm{a}_{\mathrm{n}} \times\left(\mathrm{Ll}_{\mathrm{n}}+\mathrm{Wl}_{\mathrm{n}}\right)$ \\
\hline 10 & $\mathrm{LA}=\left[\mathrm{a}_{1} \times(\mathrm{L}+\mathrm{W})\right]+\mathrm{a}_{2}$ & $28 \mathrm{LA}=\mathrm{a}_{1} \times\left(\mathrm{Ll}_{1} \times \mathrm{Wl}_{1}\right)+\ldots+\mathrm{a}_{\mathrm{n}} \times\left(\mathrm{Ll}_{\mathrm{n}} \times \mathrm{Wl} \mathrm{l}_{\mathrm{n}}\right)$ \\
\hline 11 & $\mathrm{LA}=\mathrm{a}_{1} \times\left[(\mathrm{L} \times \mathrm{W})^{\mathrm{b}}\right]$ & $29 \mathrm{LA}=\mathrm{a}_{1} \times($ Lla + Wla $)$ \\
\hline 12 & $\mathrm{LA}=\mathrm{a}_{1} \times\left[(\mathrm{L}+\mathrm{W})^{\mathrm{b}}\right]$ & $30 \mathrm{LA}=\left[\mathrm{a}_{1} \times(\mathrm{Lla}+\mathrm{Wla})\right]+\mathrm{a}_{2}$ \\
\hline & $\mathrm{LA}=\left(\mathrm{a}_{1} \times \mathrm{Ll}_{1}\right)+\ldots+\left(\mathrm{a}_{\mathrm{n}} \times \mathrm{Ll}_{\mathrm{n}}\right)$ & $31 \quad \mathrm{LA}=\mathrm{a}_{1} \times\left[(\mathrm{Lla}+\mathrm{Wla})^{\mathrm{b}}\right]$ \\
\hline 14 & $\mathrm{LA}=\mathrm{a}_{1} \times\left(\mathrm{Ll}_{1}+\ldots+\mathrm{Ll}_{\mathrm{n}}\right)$ & $32 \mathrm{LA}=\left[\mathrm{a}_{1} \times(\mathrm{Lla}+\mathrm{Wla})\right]+\left\{\mathrm{a}_{2} \times\left[(\mathrm{Lla}+\mathrm{Wla})^{2}\right]\right\}$ \\
\hline 15 & $\mathrm{LA}=\left[\mathrm{a}_{1} \times\left(\mathrm{Ll}_{1}+\ldots+\mathrm{L} \mathrm{l}_{\mathrm{n}}\right)\right]+\mathrm{a}_{2}$ & 33 LA $=a_{1} \times$ Lla $\times$ Wla \\
\hline 16 & $\mathrm{LA}=\mathrm{a}_{1} \times\left[\left(\mathrm{Ll}_{1}+\ldots+\mathrm{Ll} \mathrm{l}_{\mathrm{n}}\right)^{\mathrm{b}}\right]$ & $34 \mathrm{LA}=\left(\mathrm{a}_{1} \times \mathrm{Lla} \times \mathrm{Wla}\right)+\mathrm{a}_{2}$ \\
\hline 17 & $\mathrm{LA}=\mathrm{a}_{1} \times \mathrm{Lla}$ & $35 \mathrm{LA}=\mathrm{a}_{1} \times\left[(\mathrm{Lla} \times \mathrm{Wla})^{\mathrm{b}}\right]$ \\
\hline & $\mathrm{LA}=\left(\mathrm{a}_{1} \times \mathrm{Lla}\right)+\mathrm{a}_{2}$ & $36 \mathrm{LA}=\left(\mathrm{a}_{1} \times\right.$ Lla $\times$ Wla $)+\left\{\mathrm{a}_{2} \times\left[(\text { Lla } \times \text { Wla })^{2}\right]\right\}$ \\
\hline \multicolumn{3}{|c|}{ Analytical models based on dry mass } \\
\hline \multicolumn{3}{|c|}{$37 \quad \mathrm{LA}=\mathrm{a}_{1} \times \mathrm{DM}$} \\
\hline \multicolumn{3}{|c|}{$38 \mathrm{LA}=\left(\mathrm{a}_{1} \times \mathrm{DM}\right)+\mathrm{a}_{2}$} \\
\hline \multicolumn{3}{|c|}{$39 \quad \mathrm{LA}=\mathrm{a}_{1} \times\left(\mathrm{DM}^{\mathrm{b}}\right)$} \\
\hline \multicolumn{3}{|c|}{$40 \quad \mathrm{LA}=\left(\mathrm{a}_{1} \times \mathrm{DM}\right)+\mathrm{a}_{2} \times\left(\mathrm{DM}^{2}\right)$} \\
\hline
\end{tabular}

Were destined $70 \%$ of the data for generating models (calculation of adjusted coefficients), while the remaining aided validation and subsequent calculation of statistical indicators.

Data homogeneity was verified between the F-test and leaf area values. Normality tests were performed with independent variables (isolated linear measurements of length, width, and dry mass) and real leaf area (dependent variable) for each species. In all cases, the behavior of normal distributions was observed. For the model generation, a linear regression was constructed considering the leaf area as a dependent variable and linear (L, W, Ll, and $\mathrm{Wl}$ ) and non-linear (DM) parameters as independent variables. The Microsoft Excel Solver tool was used and the adjusted coefficients from regressions were determined by maximizing the coefficient of determination $\left(\mathrm{R}^{2}\right)$.
In order to assess the performance of generated models in the leaf area estimation, the following statistical indicators were calculated: MBE (mean bias error), RMSE (root mean square error), $\mathrm{d}$ (Willmott adjustment index), and $\mathrm{c}$ (performance index) (WILLMOTT, 1981; LEITE; ANDRADE, 2002).

$$
\begin{aligned}
& \mathrm{MBE}=\sum_{\mathrm{i}=1}^{1}(\mathrm{Ei}-\mathrm{Oi}) / \mathrm{n} \\
& \mathrm{RMSE}=\left[\sum_{\mathrm{i}=1}^{1}(\mathrm{Ei}-\mathrm{Oi})^{2} / \mathrm{n}\right] \\
& \mathrm{d}=1-\left(\sum_{\mathrm{i}=1}^{\mathrm{n}}(\mathrm{Ei}-\mathrm{Oi})^{2} /\left[\sum_{\mathrm{i}=1}^{\mathrm{n}}(|\mathrm{Ei}-\overline{\mathrm{O}}|)+(\mid \mathrm{Oi}-\overline{\mathrm{O}} \mathrm{l})^{2}\right]\right) \\
& \mathrm{c}=\mathrm{r} \mathrm{x} \mathrm{dw} \\
& \mathrm{r}=\sum_{\mathrm{i}=1}^{\mathrm{n}}(\mathrm{Oi}-\overline{\mathrm{O}})(\mathrm{Ei}-\overline{\mathrm{E}}) /\left\{\left[\sum_{\mathrm{i}=1}^{\mathrm{n}}(\mathrm{Oi}-\overline{\mathrm{O}})^{2}\right]\left[\sum_{\mathrm{i}=1}^{\mathrm{n}}(\mathrm{Ei}-\overline{\mathrm{E}})^{2}\right]\right\}
\end{aligned}
$$

Where Ei - estimated value; Oi - observed value; $\mathrm{n}$ - number of observations; $\overline{\mathrm{O}}$ - average of observed values; $\overline{\mathrm{E}}$ - average of estimated values; and $\mathrm{r}$ - correlation coefficient. 
Considering the statistical indicators and aiming at analyzing the performance of models, the weighted value of statistical indicator (Wv) method was used according to Thiersch (1997). For Wv acquisition, weights from 1 to " $n$ " were assigned for each statistical indicator at each model, where " $n$ " is the number of models tested (36 for linear measurements and 4 for dry mass). Thus, the best model was the one that presented the lowest sum of weights (a low accumulated Wv).

\section{RESULTS}

The values of leaf area, linear measurements and dry mass are shown in Table 2. T. impetiginosa presented the highest average values of Lp ( $\mathrm{L}$ with petiole), W, LA, and DM (with and without petiole) whereas $H$. chrysotrichus presented the lowest values of linear measurements, LA, and DM (with and without petiole). $T$. roseoalba presented the highest average of $\mathrm{L}$ without petiole and the lowest average of Lp. Regarding the leaflets, the highest average values for $\mathrm{Ll}$ were observed in $T$. impetiginosa and for $\mathrm{Wl}$ in $T$. roseoalba. The lowest averages of leaflet linear measurements were observed in $H$. chrysotrichus.

The linear regressions calculated between LA area and DM without and with petiole (Figure 2) presented high coefficients of determination $\left(\mathrm{R}^{2}\right.$ between 0.99555 and 0.99994 ), suggesting that the presence of petiole had no considerable influence on these parameters. For this reason, all assessed models in this study are related only to values disregarding the petiole in length, leaf area, and dry mass measurements due to its low influence on these parameters.

Table 2. Average values $(\overline{\mathbf{x}})$ and standard deviations $(\sigma)$ for the parameters leaf area (LA), leaf length (L), petiole length (Lp), dry mass (DM), leaflet length (Ll), leaf width (W), and leaflet width (Wl) obtained for leaves used for generating and validating mathematical models of leaf area estimation.

\begin{tabular}{ccccccc} 
& \multicolumn{3}{c}{ Generation of models } & \multicolumn{3}{c}{ Validation of models } \\
\cline { 2 - 7 } & $\begin{array}{c}\text { Tabebuia } \\
\text { impetiginosa }\end{array}$ & $\begin{array}{c}\text { Tabebuia } \\
\text { roseoalba }\end{array}$ & $\begin{array}{c}\text { Handroanthus } \\
\text { crysotrichus }\end{array}$ & $\begin{array}{c}\text { Tabebuia } \\
\text { impetiginosa }\end{array}$ & $\begin{array}{c}\text { Tabebuia } \\
\text { roseoalba }\end{array}$ & $\begin{array}{c}\text { Handroanthus } \\
\text { crysotrichus }\end{array}$ \\
\hline$\overline{\mathrm{x}}$ & 309.50 & 145.00 & 68.10 & 63.30 & 72.90 & 46.00 \\
$\sigma$ & 279.90 & 64.50 & 32.40 & 61.80 & 79.70 & 28.70 \\
\hline$\overline{\mathrm{x}}$ & 36.10 & 27.40 & 14.60 & 17.60 & 18.90 & 11.40 \\
$\sigma$ & 16.00 & 7.20 & 3.60 & 11.50 & 7.60 & 4.50 \\
\hline$\overline{\mathrm{x}}$ & 15.00 & 2.20 & 4.60 & 13.00 & 2.70 & 3.80 \\
$\sigma$ & 7.40 & 1.10 & 1.40 & 8.00 & 1.10 & 1.40 \\
\hline$\overline{\mathrm{x}}$ & 3.570 & 1.582 & 0.82 & 2.436 & 3.976 & 0.534 \\
$\sigma$ & 3.545 & 0.931 & 0.468 & 3.877 & 1.021 & 0.337 \\
\hline$\overline{\mathrm{x}}$ & 302.20 & 142.90 & 49.40 & 124.80 & 119.80 & 52.50 \\
$\sigma$ & 274.90 & 47.00 & 26.04 & 57.30 & 47.00 & 30.10 \\
\hline$\overline{\mathrm{x}}$ & 20.80 & 24.10 & 8.30 & 17.90 & 22.10 & 8.10 \\
$\sigma$ & 9.50 & 4.80 & 2.50 & 5.20 & 4.80 & 2.70 \\
\hline$\overline{\mathrm{x}}$ & 2.971 & 1.385 & 0.745 & 2.207 & 1.731 & 0.495 \\
$\sigma$ & 3.477 & 0.854 & 0.433 & 3.679 & 0.918 & 0.316 \\
\hline$\overline{\mathrm{x}}$ & 16.20 & 10.30 & 5.20 & 12.40 & 9.80 & 4.90 \\
$\sigma$ & 9.30 & 2.40 & 2.50 & 5.40 & 2.40 & 2.30 \\
\hline$\overline{\mathrm{x}}$ & 36.20 & 19.00 & 9.70 & 28.00 & 17.70 & 10.20 \\
$\sigma$ & 16.90 & 4.00 & 3.00 & 5.40 & 4.00 & 3.50 \\
\hline$\overline{\mathrm{x}}$ & 5.40 & 6.30 & 2.80 & 3.80 & 5.70 & 2.90 \\
$\sigma$ & 3.00 & 1.60 & 1.40 & 1.30 & 1.60 & 1.00 \\
\hline & & & & & &
\end{tabular}



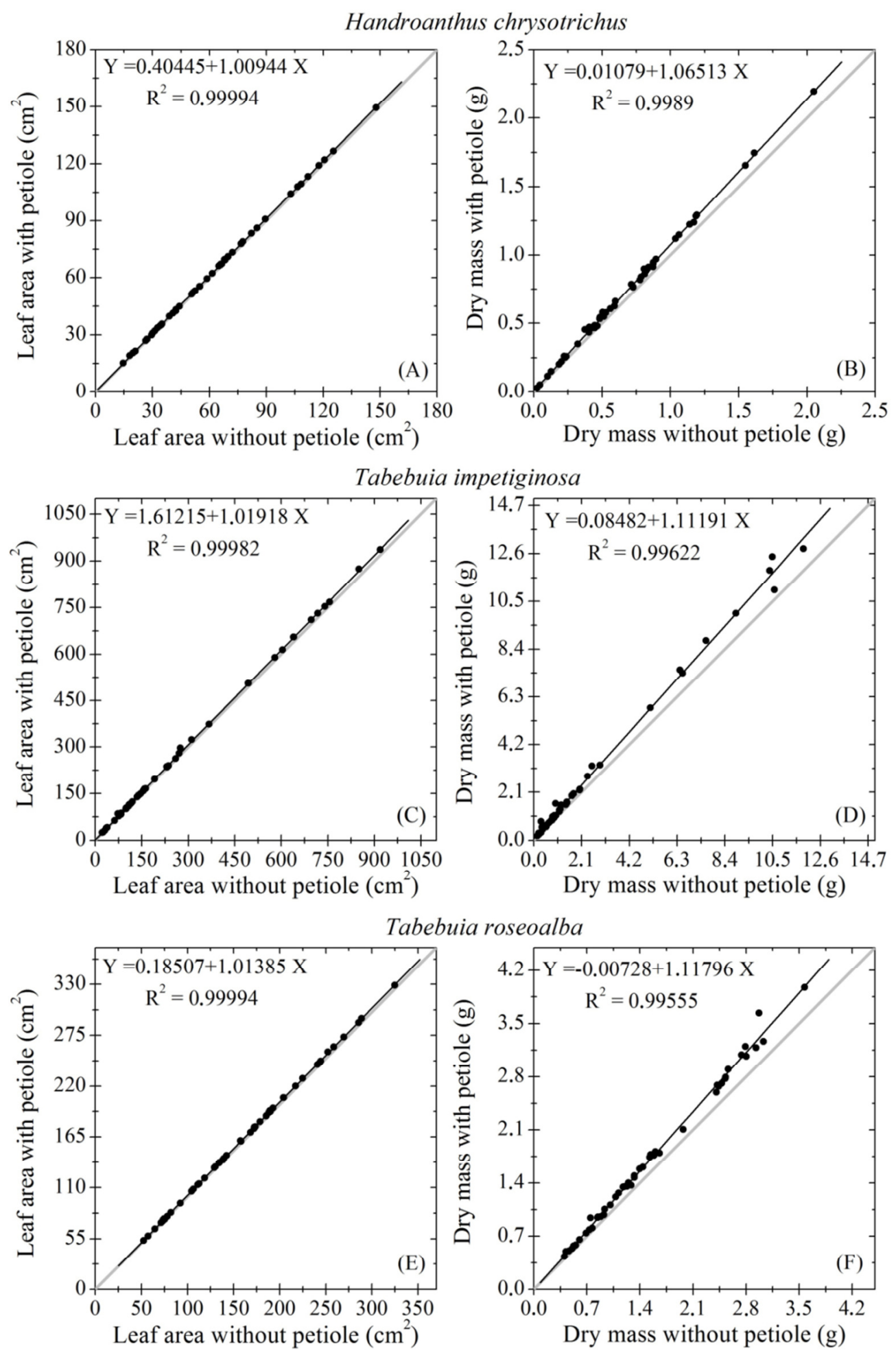

Figure 2. Linear correlations between leaf area and dry mass without and with petiole measured in leaves of $H$. chrysotrichus (A and B), T. impetiginosa (C and D) and T. roseoalba (E and F).

The regression analyses of the generated models and their respective validations (statistical parameters) are presented in Tables 3, 4, and 5 respectively for $H$. chrysotrichus, $T$. impetiginosa, and $T$. roseoalba. Thus, the two best models for each species were analyzed considering the linear measurements and dry mass (models with lower accumulated weighted value).

The best models based on linear measurements were those of numbers 8 and 12 ( $\sum \mathrm{Wv}$ of 16 and 18 , respectively) for $H$. chrysotrichus, whereas for species from the genus Tabebuia, the best ones were the models number 26 and 23, which presented accumulated Wv 
respectively of 4 and 8 for $T$. impetiginosa and 10 for T. roseoalba.

Regarding the models based on dry mass, $T$. roseoalba presented a lower accumulated $\mathrm{Wv}$ in the models number 37 ( $\sum \mathrm{Wv}_{\mathrm{v}}$ of 7$)$ and $38\left(\sum \mathrm{Wv}\right.$ of 8$)$ whereas $H$. chrysotrichus and $T$. impetiginosa presented the lowest accumulated $\mathrm{Wv}$ for the models 39 ( $\sum \mathrm{Wv}$ of 8 and 9 , respectively) and 40 ( $\sum \mathrm{Wv}$ of 8 and 5 , respectively).

Table 3. Adjusted coefficients $\left(\mathrm{a}_{\mathrm{n}}\right.$ and $\left.\mathrm{b}\right)$, coefficients of determination $\left(\mathrm{R}^{2}\right)$, and statistical indicators for models based on linear measurements or dry mass of leaf area estimation of Handroanthus chrysotrichus.

\begin{tabular}{|c|c|c|c|c|c|c|c|c|c|c|c|c|}
\hline \multirow{2}{*}{ Model } & \multicolumn{6}{|c|}{ Adjusted Coefficients } & \multirow{2}{*}{$\mathbf{R}^{2}$} & \multicolumn{5}{|c|}{ Estatistical validation } \\
\hline & $\mathbf{a}_{1}$ & $\mathbf{a}_{2}$ & $\mathbf{a}_{3}$ & $\mathbf{a}_{4}$ & $\mathbf{a}_{5}$ & $\mathbf{b}$ & & MBE & RMSE & d & c & $\sum \mathbf{W v}$ \\
\hline \multicolumn{13}{|c|}{ Analytic models based on linear measures } \\
\hline 1 & 6.064 & - & - & - & - & - & 0.445 & $-3.46(16)$ & $21.42(36)$ & $0.77(32)$ & $0.56(36)$ & 120 \\
\hline 2 & 7.146 & -9.739 & - & - & - & - & 0.457 & $-4.44(19)$ & $21.07(33)$ & $0.81(23)$ & $0.59(33)$ & 108 \\
\hline 3 & 3.368 & - & - & - & - & 1.262 & 0.463 & $-4.51(20)$ & 20.95 & $0.81(22)$ & $0.60(27)$ & 101 \\
\hline 4 & 5.163 & - & - & - & - & - & 0.420 & $-0.05(1)$ & $19.28(13)$ & $0.83(16)$ & $0.65(20)$ & 50 \\
\hline 5 & 5.610 & -4.732 & - & - & - & - & 0.422 & $-0.23(2)$ & $18.90(6)$ & $0.85(11)$ & $0.66(17)$ & 36 \\
\hline 6 & 3.133 & - & - & - & - & 1.207 & 0.431 & $-0.24(3)$ & $18.43(3)$ & $0.86(7)$ & $0.68(12)$ & 25 \\
\hline 7 & 0.530 & - & - & - & - & - & 0.410 & $-4.53(21)$ & $19.51(14)$ & 0.89 (1) & $0.71(3)$ & 39 \\
\hline 8 & 0.383 & 16.586 & - & - & - & - & 0.507 & $-1.26(6)$ & $18.22(1)$ & $0.87(5)$ & $0.69(4)$ & 16 \\
\hline 9 & 2.813 & - & - & - & - & - & 0.467 & $-1.17(5)$ & $19.69(16)$ & $0.81(21)$ & $0.64(21)$ & 63 \\
\hline 10 & 3.502 & -13.372 & - & - & - & - & 0.487 & $-1.97(8)$ & $18.80(5)$ & $0.86(9)$ & 0.67 (13) & 35 \\
\hline 11 & 2.476 & - & - & - & - & 0.679 & 0.495 & $-2.00(9)$ & $18.49(4)$ & $0.87(6)$ & $0.68(8)$ & 27 \\
\hline 12 & 0.922 & - & - & - & - & 1.370 & 0.499 & $-1.85(7)$ & $18.32(2)$ & 0.87 (4) & $0.69(5)$ & 18 \\
\hline 13 & 3.206 & 1.248 & 0.155 & 4.044 & 2.147 & - & 0.474 & $-5.06(23)$ & $20.34(23)$ & $0.79(24)$ & $0.64(22)$ & 92 \\
\hline 14 & 1.917 & - & - & - & - & - & 0.464 & $-5.95(27)$ & 20.83 & $0.78(28)$ & $0.62(26)$ & 108 \\
\hline 15 & 2.283 & -10.443 & - & - & - & - & 0.478 & $-7.51(32)$ & $20.34(22)$ & $0.82(20)$ & 0.65 (19) & 93 \\
\hline 16 & 0.670 & - & - & - & - & 1.309 & 0.489 & $-7.95(34)$ & $20.10(19)$ & 0.83 (18) & $0.67(15)$ & 86 \\
\hline 17 & 9.587 & - & - & - & - & - & 0.4645 & $-5.95(26)$ & $20.83(26)$ & $0.78(27)$ & $0.62(25)$ & 104 \\
\hline 18 & 11.415 & -10.443 & - & - & - & - & 0.478 & $-7.51(31)$ & $20.34(21)$ & 0.82 (19) & 0.65 (18) & 89 \\
\hline 19 & 5.508 & - & - & - & - & 1.309 & 0.489 & $-7.95(33)$ & 20.10 & $0.83(17)$ & $0.67(14)$ & 82 \\
\hline 20 & 8.834 & -3.141 & 5.157 & 7.066 & 0.393 & - & 0.468 & $-0.96(4)$ & $20.71(25)$ & 0.78 (29) & $0.59(28)$ & 86 \\
\hline 21 & 3.483 & - & - & - & - & - & 0.391 & $-2.70(15)$ & $20.89(29)$ & $0.77(31)$ & $0.59(30)$ & 105 \\
\hline 22 & 3.471 & 0.196 & - & - & - & - & 0.391 & $-2.68(13)$ & $20.91(31)$ & $0.77(34)$ & $0.59(32)$ & 110 \\
\hline 23 & 4.154 & - & - & - & - & 0.937 & 0.394 & $-2.38(10)$ & $21.21(34)$ & $0.76(35)$ & $0.58(34)$ & 113 \\
\hline 24 & 17.416 & - & - & - & - & - & 0.391 & $-2.70(14)$ & $20.89(28)$ & $0.77(30)$ & $0.59(29)$ & 101 \\
\hline 25 & 17.354 & 0.196 & - & - & - & - & 0.391 & $-2.68(12)$ & $20.91(30)$ & $0.77(33)$ & $0.59(31)$ & 106 \\
\hline 26 & 18.775 & - & - & - & - & 0.937 & 0.394 & $-2.38(11)$ & $21.21(35)$ & $0.76(36)$ & $0.58(35)$ & 117 \\
\hline 27 & 2.385 & -0.004 & 0.897 & 2.764 & 0.447 & - & 0.487 & $-3.67(17)$ & $20.30(20)$ & $0.79(25)$ & $0.63(23)$ & 85 \\
\hline 28 & 1.270 & 0.085 & 0.483 & 0.978 & 0.110 & - & 0.473 & $-8.35(35)$ & $19.15(10)$ & $0.88(2)$ & $0.72(1)$ & 48 \\
\hline 29 & 6.240 & - & - & - & - & - & 0.476 & $-4.36(18)$ & $20.47(24)$ & $0.79(26)$ & $0.63(24)$ & 92 \\
\hline 30 & 7.688 & -12.699 & - & - & - & - & 0.494 & $-5.88(25)$ & $19.64(15)$ & $0.83(15)$ & $0.66(16)$ & 71 \\
\hline 31 & 2.906 & - & - & - & - & 1.344 & 0.506 & $-6.18(29)$ & $19.26(12)$ & $0.84(12)$ & $0.68(11)$ & 64 \\
\hline 32 & 4.002 & 0.238 & - & - & - & - & 0.509 & $-6.08(28)$ & $19.20(11)$ & $0.84(13)$ & $0.68(9)$ & 61 \\
\hline 33 & 2.820 & - & - & - & - & - & 0.401 & $-9.72(36)$ & $19.74(17)$ & $0.87(3)$ & $0.71(2)$ & 58 \\
\hline 34 & 2.012 & 17.193 & - & - & - & - & 0.509 & $-4.79(22)$ & $18.92(7)$ & $0.84(14)$ & $0.69(6)$ & 49 \\
\hline 35 & 7.986 & - & - & - & - & 0.668 & 0.504 & $-5.25(24)$ & $19.05(8)$ & $0.85(10)$ & $0.68(10)$ & 52 \\
\hline 36 & 3.765 & -0.035 & - & - & - & - & 0.489 & $-6.43(30)$ & $19.15(9)$ & $0.86(8)$ & $0.69(7)$ & 54 \\
\hline \multicolumn{13}{|c|}{ Analytic models based on dry mass } \\
\hline 37 & 80.411 & - & - & - & - & - & 0.292 & $-5.33(2)$ & $30.52(3)$ & $0.59(3)$ & $0.20(3)$ & 11 \\
\hline 38 & 51.230 & 28.882 & - & - & - & - & 0.492 & $9.12(4)$ & $28.17(1)$ & $0.54(4)$ & $0.18(4)$ & 13 \\
\hline 39 & 82.438 & - & - & - & - & 0.569 & 0.502 & $6.53(3)$ & $29.07(2)$ & $0.61(2)$ & $0.23(1)$ & 8 \\
\hline 40 & 119.510 & -32.044 & - & - & - & - & 0.474 & $3.19(1)$ & $31.19(4)$ & $0.62(1)$ & $0.22(2)$ & 8 \\
\hline
\end{tabular}

Weighted (in parenthesis) and accumulated $(\Sigma \mathrm{Wv})$ values; MBE: mean bias error $\left(\mathrm{cm}^{2}\right)$; RMSE: root mean square error $(\mathrm{cm})$; $\mathrm{d}$ : Willmott adjustment index, and c: performance index. 
Table 4. Adjusted coefficients ( $\mathrm{a}_{\mathrm{n}}$ and $\left.\mathrm{b}\right)$, coefficients of determination $\left(\mathrm{R}^{2}\right)$, and statistical indicators for models based on linear measurements or dry mass of leaf area estimation of Tabebuia impetiginosa.

\begin{tabular}{|c|c|c|c|c|c|c|c|c|c|c|c|c|}
\hline \multirow{2}{*}{ Model } & \multicolumn{6}{|c|}{ Adjusted Coefficients } & \multirow{2}{*}{$\mathbf{R}^{2}$} & \multicolumn{5}{|c|}{ Estatistical validation } \\
\hline & $\mathbf{a}_{1}$ & $\mathbf{a}_{2}$ & $\mathbf{a}_{3}$ & $\mathbf{a}_{4}$ & $\mathbf{a}_{5}$ & b & & MBE & RMSE & d & c & $\sum \mathbf{W v}$ \\
\hline \multicolumn{13}{|c|}{ Analytic models based on linear measures } \\
\hline $\mathbf{1}$ & 16.495 & - & - & - & - & - & 0.685 & $169.99(36)$ & $178.03(36)$ & $0.42(33)$ & $0.33(33)$ & 138 \\
\hline 3 & 0.764 & - & - & - & - & 1.921 & 0.828 & $83.11(27)$ & $109.94(24)$ & $0.64(24)$ & $0.51(26)$ & 101 \\
\hline 4 & 9.553 & - & - & - & - & - & 0.738 & $142.57(32)$ & $144.79(34)$ & $0.46(31)$ & $0.41(31)$ & 128 \\
\hline 7 & 0.346 & - & - & - & - & - & 0.907 & $54.47(23)$ & 62.99 (19) & $0.81(21)$ & $0.76(20)$ & 83 \\
\hline 8 & 0.358 & -17.079 & - & - & - & - & 0.908 & $43.39(17)$ & $54.92(16)$ & $0.85(16)$ & $0.79(16)$ & 65 \\
\hline 9 & 6.093 & - & - & - & - & - & 0.732 & $154.62(35)$ & $156.42(35)$ & $0.45(32)$ & $0.41(32)$ & 134 \\
\hline 10 & 10.008 & - & - & - & - & - & 0.890 & $66.55(25)$ & $82.78(23)$ & $0.75(23)$ & $0.69(23)$ & 94 \\
\hline 11 & 0.252 & - & - & - & - & 1.042 & 0.908 & $46.36(19)$ & $55.72(18)$ & $0.84(17)$ & $0.79(17)$ & 71 \\
\hline 15 & 7.086 & - & - & - & - & - & 0.943 & 44.67 (18) & $64.31(20)$ & $0.83(18)$ & 0.77 (18) & 74 \\
\hline 16 & 0.040 & - & - & - & - & 1.992 & 0.967 & 29.78 (13) & $42.76(13)$ & 0.89 (13) & $0.82(14)$ & 53 \\
\hline 17 & 21.595 & - & - & - & - & - & 0.783 & $143.13(34)$ & $144.65(33)$ & $0.49(30)$ & $0.45(29)$ & 126 \\
\hline 18 & 34.777 & - & - & - & - & - & 0.943 & $47.12(20)$ & $64.97(21)$ & 0.82 (19) & 0.77 (19) & 79 \\
\hline 19 & 0.981 & - & - & - & - & 1.991 & 0.967 & $29.77(12)$ & $42.75(12)$ & $0.89(12)$ & $0.82(13)$ & 49 \\
\hline 20 & 87.262 & 27.604 & -68.319 & 77.911 & -53.329 & - & 0.927 & $40.14(15)$ & $115.69(25)$ & $0.33(34)$ & $0.05(34)$ & 108 \\
\hline 21 & 12.769 & - & - & - & - & - & 0.831 & $117.67(29)$ & $118.94(26)$ & $0.53(26)$ & $0.52(24)$ & 105 \\
\hline 22 & 19.077 & - & - & - & - & - & 0.960 & $21.13(11)$ & $27.00(9)$ & $0.95(9)$ & $0.92(9)$ & 38 \\
\hline 23 & 0.553 & - & - & - & - & 1.853 & 0.981 & $7.89(2)$ & $16.13(2)$ & $0.97(2)$ & $0.95(2)$ & 8 \\
\hline 24 & 63.845 & - & - & - & - & - & 0.831 & $117.67(30)$ & $118.94(27)$ & $0.53(27)$ & $0.52(25)$ & 109 \\
\hline 25 & 95.386 & - & - & - & - & - & 0.960 & $21.13(10)$ & $27.00(8)$ & $0.95(8)$ & $0.92(8)$ & 34 \\
\hline 32 & -0.109 & 0.533 & - & - & - & - & 0.981 & $19.19(8)$ & $29.46(10)$ & $0.94(10)$ & $0.89(10)$ & 38 \\
\hline 33 & 2.782 & - & - & - & - & - & 0.988 & $10.82(3)$ & $18.67(3)$ & $0.97(3)$ & $0.94(4)$ & 13 \\
\hline 34 & 2.774 & 1.532 & - & - & - & - & 0.988 & $11.96(4)$ & $19.32(4)$ & $0.97(4)$ & $0.94(5)$ & 17 \\
\hline 35 & 2.993 & - & - & - & - & 0.986 & 0.988 & $13.52(7)$ & $20.34(5)$ & $0.97(7)$ & $0.93(7)$ & 26 \\
\hline 36 & 2.852 & - & - & - & - & - & 0.988 & $13.43(5)$ & $20.36(6)$ & $0.97(6)$ & $0.93(6)$ & 23 \\
\hline \multicolumn{13}{|c|}{ Analytic models based on dry mass } \\
\hline 37 & 85.025 & - & - & - & - & - & 0.887 & $62.85(1)$ & $259.78(4)$ & $0.48(4)$ & $0.44(4)$ & 13 \\
\hline 38 & 75.389 & 66.849 & - & - & - & - & 0.920 & $108.44(4)$ & $243.44(3)$ & $0.48(3)$ & $0.44(3)$ & 13 \\
\hline 39 & 153.802 & - & - & - & - & 0.718 & 0.946 & $105.38(3)$ & $229.61(2)$ & $0.50(2)$ & $0.46(2)$ & 9 \\
\hline 40 & 143.610 & -6.383 & - & - & - & - & 0.974 & $80.41(2)$ & $205.28(1)$ & $0.54(1)$ & $0.50(1)$ & 5 \\
\hline
\end{tabular}


Table 5. Adjusted coefficients $\left(a_{n}\right.$ and $\left.b\right)$, coefficients of determination $\left(R^{2}\right)$, and statistical indicators for models based on linear measurements or dry mass of leaf area estimation of Tabebuia roseoalba.

\begin{tabular}{|c|c|c|c|c|c|c|c|c|c|c|}
\hline \multirow{2}{*}{ Model } & \multicolumn{4}{|c|}{ Adjusted Coefficients } & \multirow{2}{*}{$\mathbf{R}^{2}$} & \multicolumn{5}{|c|}{ Estatistical validation } \\
\hline & $\mathbf{a}_{1}$ & $\mathbf{a}_{2}$ & $\mathbf{a}_{3}$ & $\mathbf{b}$ & & MBE & RMSE & d & c & $\sum \mathbf{W v}$ \\
\hline \multicolumn{11}{|c|}{ Analytic models based on linear measures } \\
\hline 1 & 6.013 & - & - & - & 0.586 & $13.17(26)$ & $38.29(19)$ & $0.73(32)$ & $0.47(32)$ & 109 \\
\hline 2 & 6.903 & -23.183 & - & - & 0.597 & $9.67(22)$ & $37.37(9)$ & $0.76(25)$ & $0.49(22)$ & 78 \\
\hline 3 & 2.931 & - & - & 1.218 & 0.603 & $8.17(18)$ & $37.23(8)$ & $0.77(24)$ & $0.49(23)$ & 73 \\
\hline 4 & 7.735 & - & - & - & 0.668 & $17.34(33)$ & 41.09 (29) & $0.72(34)$ & $0.44(36)$ & 132 \\
\hline 5 & 10.628 & -58.598 & - & - & 0.724 & $10.05(23)$ & $41.02(28)$ & $0.77(21)$ & $0.47(31)$ & 103 \\
\hline 6 & 1.446 & - & - & 1.550 & 0.747 & $7.52(17)$ & $41.33(30)$ & $0.77(23)$ & $0.46(33)$ & 103 \\
\hline 7 & 0.278 & - & - & - & 0.640 & $-6.11(12)$ & $41.00(27)$ & 0.79 (14) & $0.49(18)$ & 71 \\
\hline 8 & 0.215 & 38.571 & - & - & 0.717 & $6.55(13)$ & $37.76(14)$ & $0.77(22)$ & $0.48(26)$ & 75 \\
\hline 9 & 3.401 & - & - & - & 0.654 & $15.73(32)$ & $38.88(25)$ & $0.73(31)$ & $0.48(28)$ & 116 \\
\hline 10 & 4.530 & -51.988 & - & - & 0.700 & 8.73 (19) & $37.44(12)$ & 0.79 (13) & $0.51(13)$ & 57 \\
\hline 11 & 1.670 & - & - & 0.723 & 0.714 & $7.14(16)$ & $37.71(13)$ & 0.79 (16) & $0.50(17)$ & 62 \\
\hline 12 & 0.628 & - & - & 1.436 & 0.710 & $6.91(15)$ & $37.42(11)$ & $0.79(15)$ & $0.50(16)$ & 57 \\
\hline 13 & -0.313 & 4.448 & 10.985 & - & 0.661 & $14.50(30)$ & $38.58(24)$ & $0.75(26)$ & $0.48(25)$ & 105 \\
\hline 14 & 4.778 & - & - & - & 0.632 & $20.02(35)$ & $41.68(33)$ & $0.71(35)$ & $0.44(34)$ & 137 \\
\hline 15 & 7.667 & -93.625 & - & - & 0.741 & $10.91(24)$ & $41.61(32)$ & 0.79 (17) & $0.49(20)$ & 93 \\
\hline 16 & 0.321 & - & - & 1.768 & 0.759 & $9.34(21)$ & $41.73(35)$ & $0.78(20)$ & $0.47(29)$ & 105 \\
\hline 17 & 14.335 & - & - & - & 0.632 & $20.02(36)$ & $41.68(34)$ & $0.71(36)$ & $0.44(35)$ & 141 \\
\hline 18 & 23.001 & -93.624 & - & - & 0.741 & $10.91(25)$ & $41.61(31)$ & 0.79 (18) & $0.49(21)$ & 95 \\
\hline 19 & 2.238 & - & - & 1.768 & 0.759 & $9.34(20)$ & $41.74(36)$ & 0.78 (19) & $0.47(30)$ & 105 \\
\hline 20 & 7.338 & 3.662 & 12.390 & - & 0.719 & $14.06(29)$ & $38.05(16)$ & $0.74(30)$ & $0.48(24)$ & 99 \\
\hline 21 & 7.801 & - & - & - & 0.714 & $13.86(27)$ & $37.22(6)$ & $0.75(28)$ & $0.51(14)$ & 75 \\
\hline 22 & 11.999 & -84.345 & - & - & 0.820 & $1.45(5)$ & $35.97(4)$ & $0.82(1)$ & $0.56(1)$ & 11 \\
\hline 23 & 0.975 & - & - & 1.684 & 0.837 & $-0.49(2)$ & $35.69(2)$ & $0.82(3)$ & $0.55(3)$ & 10 \\
\hline 24 & 23.403 & - & - & - & 0.714 & $13.86(28)$ & $37.22(7)$ & $0.75(27)$ & $0.51(15)$ & 77 \\
\hline 25 & 35.998 & -84.345 & - & - & 0.820 & $1.45(6)$ & $35.97(3)$ & $0.82(2)$ & $0.56(2)$ & 13 \\
\hline 26 & 6.196 & - & - & 1.684 & 0.837 & $-0.49(1)$ & $35.69(1)$ & $0.82(4)$ & $0.55(4)$ & 10 \\
\hline 27 & 0.961 & 2.305 & 5.991 & - & 0.688 & $14.65(31)$ & $38.15(17)$ & $0.74(29)$ & 0.49 (19) & 96 \\
\hline 28 & 0.721 & 0.710 & 0.588 & - & 0.825 & $1.01(4)$ & $38.38(21)$ & $0.81(9)$ & $0.53(10)$ & 44 \\
\hline 29 & 8.905 & - & - & - & 0.673 & $17.92(34)$ & $39.57(26)$ & $0.73(33)$ & $0.48(27)$ & 120 \\
\hline 30 & 14.501 & -97.752 & - & - & 0.797 & $6.69(14)$ & $38.43(22)$ & $0.81(8)$ & $0.53(6)$ & 50 \\
\hline 31 & 0.840 & - & - & 1.814 & 0.820 & $4.50(10)$ & $38.31(20)$ & $0.81(11)$ & $0.52(11)$ & 52 \\
\hline 32 & 1.654 & 0.398 & - & - & 0.821 & $4.66(11)$ & $38.21(18)$ & $0.80(12)$ & $0.52(12)$ & 53 \\
\hline 33 & 2.058 & - & - & - & 0.823 & $-0.85(3)$ & $38.43(23)$ & $0.81(6)$ & $0.53(8)$ & 40 \\
\hline 34 & 1.878 & 14.677 & - & - & 0.832 & $3.42(9)$ & $37.19(5)$ & $0.81(10)$ & $0.53(9)$ & 33 \\
\hline 35 & 3.181 & - & - & - & 0.831 & $3.16(8)$ & $37.38(10)$ & $0.81(7)$ & $0.53(7)$ & 32 \\
\hline 36 & 2.252 & -0.002 & - & - & 0.829 & $2.37(7)$ & $37.79(15)$ & $0.81(5)$ & $0.54(5)$ & 32 \\
\hline \multicolumn{11}{|c|}{ Analytic models based on dry mass } \\
\hline 37 & 90.623 & - & - & - & 0.319 & $-27.30(4)$ & $36.21(1)$ & $0.95(1)$ & $0.90(1)$ & 7 \\
\hline 38 & 56.757 & 64.234 & - & - & 0.594 & $-21.69(2)$ & $38.44(2)$ & $0.91(2)$ & $0.87(2)$ & 8 \\
\hline 39 & 125.490 & - & - & 0.539 & 0.599 & $-21.34(1)$ & $40.02(3)$ & $0.90(3)$ & $0.86(3)$ & 10 \\
\hline 40 & 154.653 & -28.035 & - & - & 0.600 & $-22.51(3)$ & $47.01(4)$ & $0.85(4)$ & $0.75(4)$ & 15 \\
\hline
\end{tabular}

Weighted (in parenthesis) and accumulated $(\Sigma \mathrm{Wv})$ values; MBE: mean bias error $\left(\mathrm{cm}^{2}\right)$; RMSE: root mean square error $(\mathrm{cm})$; d: Willmott adjustment index, and c: performance index.

\section{DISCUSSION}

Models based on linear measurements with the best performance of LA area estimation for $H$. chrysotrichus used the combination of leaf measurements (length and width) as independent variables, being the first model based on their multiplication (model 8) and the second model based on their sum (model 12).

Models using $\mathrm{L}$ and $\mathrm{W}$ products are the most reported in the literature since they provided better accuracy in leaf area estimation for several 
plant species. For instance we may cite hazelnut (CRISTOFORI et al., 2007), bedding plants (GIUFFRIDA et al., 2011), grapevine (TSIALTAS et al., 2008; MONTERO et al., 2000), potato (BUSATO et al., 2010), strawberry (PIRES et al., 1999; STRIK; PROCTOR, 1985), groundnut (CARDOZO et al., 2014), clary sage (KUMAR; SHARMA, 2010), among others.

Likewise, these models based on $\mathrm{L}$ and $\mathrm{W}$ products were also the most indicated for forest species such as Mangifera indica (LIMA et al., 2012), Coffea arabica (SCHMILDT et al., 2014), Combretum leprosum (CANDIDO et al., 2013), Hancornia speciosa (FONSECA; CONDÉ, 1994), Zizyphus joazeiro (MARACAJÁ et al., 2008), Citrus limonia (SILVA et al., 2013), Tabebuia aurea, Schinopsis brasiliensis (QUEIROZ et al., 2013) and Pouteria caimito (SILVA et al., 2014). In this sense, Silva et al. (2013), analysing Citrus limonia leaves, obtained a linear model similar to 8 as one of the best in the estimation. On the other hand, no models of leaf area estimation based on the sum of $\mathrm{L}$ and $\mathrm{W}$ as independent variables were found in the literature.

In contrast, the leaf area of $T$. impetiginosa and $T$. roseoalba are better estimated by potential models based only on leaflet width as an independent variable (its average value for model 26 and its sum for model 23). Studies that consider leaflet measurements for leaf area estimation are typically unavailable in the literature. For Phaseolus vulgaris, Toebe et al. (2012) concluded that one of their best models on leaf area estimation considering the width of the central leaflet $(\mathrm{Cl})$ as an independent variable was also potential $\left(\mathrm{LA}=\mathrm{a}_{1} \times \mathrm{Cl}^{\mathrm{b}}\right)$.

For the best models of $T$. impetiginosa and T. roseoalba, the measurements of all leaf and leaflets are needed, which represent an extra work compared to the measurements performed only in the leaves. Thus, the required accuracy and available time need to be considered prior to carrying out analyses of this nature.

Regarding the models based on dry mass, the best performance for $T$. roseoalba was observed for the models 37 and 38 whereas, for $H$. chrysotrichus and T. impetiginosa, the models 39 and 40 stood out. A similar model to the 39 was considered as the best in estimating leaf area in grapevine (MONTERO et al., 2000), as well as similar models to the 38 and 39, allowed a high accuracy in estimating leaf area in Eucalyptus grandis $\times$ Eucalyptus urophylla (DIAO et al., 2010).

According to Ma et al. (1992), the leaf area can be estimated by its dry mass. However, although these models present good results, their main disadvantage is the need to destroy the leaves for analysis and, consequently, a minimum structure for its acquisition is required, as a balance and greenhouse.

Regarding dry mass or linear measurements, Montero et al. (2000) emphasize that attention is needed when using only one variable in models of leaf area estimation, because despite the good results provided by them, the specific leaf area is inconstant, and changes may occur in plants due to time, phenological cycles, and environmental conditions.

\section{CONCLUSIONS}

Models based on linear measurements as an independent variable, providing the best performance of leaf area (LA) estimation for $T$. impetiginosa and $T$. roseoalba, used the average leaflet width (Wla) measurements: $\mathrm{LA}=10.919 \times \mathrm{Wla}^{1.854}$ and $\mathrm{LA}=6.196 \times \mathrm{Wla}^{1.684}$, respectively. For $H$. chrysotrichus, the model was based on the length and width of leaves ( $\mathrm{L}$ and $\mathrm{W}$ ): $\mathrm{LA}=(0.383 \times \mathrm{L} \times \mathrm{W})+16.586$.

The best models of leaf area estimation considering dry mass (DM) were $\mathrm{LA}=119.510 \times \mathrm{DM}-32.044 \times \mathrm{DM}^{2}$ for $H$. chrysotrichus, $\mathrm{LA}=143.610 \times \mathrm{DM}-6.383 \times \mathrm{DM}^{2}$ for $T$. impetiginosa, and $\mathrm{LA}=90.623 \times \mathrm{DM}$ for $T$. roseoalba.

\section{ACKNOWLEDGEMENTS}

The authors would like to thank the Coordenação de Aperfeiçoamento de Pessoal de Nível Superior (CAPES) and the Fundação de Amparo à Pesquisa do Estado de Mato Grosso (Fapemat) for the financial support to the scientific research projects of the Group Interações Ambiente e Planta.

RESUMO: A área foliar (AF) é um importante parâmetro para estudos fisiológicos e fitotécnicos, e sua obtenção de forma rápida, precisa e com baixos custos é essencial e desejável. Neste contexto, a modelagem matemática é empregada como ferramenta para estimar a AF a partir de sua relação com parâmetros biométricos e biomassa. Este estudo objetivou gerar, validar e determinar os melhores modelos de estimativa matemática de AF utilizando as variáveis lineares comprimento (com e sem pecíolo) e largura das folhas e folíolos; e a partir de massa seca das espécies nativas Tabebuia 
roseoalba, Tabebuia impetiginosa e Handroanthus chrysotrichus coletadas em Sinop, Mato Grosso (Brasil) entre janeiro e março de 2014. A avaliação dos modelos foi realizada pelo método dos valores ponderados das estimativas estatísticas. Os modelos baseados em medidas lineares como variáveis independentes que proporcionaram melhor desempenho na estimativa da AF para T. impetiginosa e $T$. roseoalba empregam a média da largura dos folíolos (Lfm): $\mathrm{AF}=10.919 \times\left(\mathrm{Lfm}^{1.854}\right)$ e $\mathrm{AF}=6.196 \times\left(\mathrm{Lfm}^{1.684}\right)$, respectivamente. Para $H$. chrysotrichus o modelo baseia-se no comprimento e largura das folhas $(\mathrm{C}$ e L): $\mathrm{AF}=(0.383 \times \mathrm{C} \times \mathrm{L})+16.586$. Os melhores modelos de estimativa de área foliar considerando massa seca (MS) foram AF=119.510 $\times \mathrm{MS}-32.044 \times \mathrm{MS}^{2}$ para H. chrysotrichus, $\mathrm{AF}=143.610 \times \mathrm{MS}-6,383 \times \mathrm{MS}^{2}$ para $T$. impetiginosa e $\mathrm{AF}=90.623 \times \mathrm{MS}$ para $T$. roseoalba.

PALAVRAS-CHAVE: Biometria. Medidas foliares. Análise de regressão. Indicador estatístico.

\section{REFERENCES}

AQUINO, L. A. de; SANTOS JUNIOR, V. C. dos; GUERRA, J. V. S.; COSTA, M. M. Estimativa da área foliar do girassol por método não destrutivo. Bragantia, Campinas, v. 70, n. 4, p. 832-836, 2011. http://dx.doi.org/10.1590/S0006-87052011000400015.

BUSATO C.; FONTES, P. C. R.; BRAUN, H.; BUSATO, C. C. M. Estimativa da área foliar da batateira, cultivar Atlantic, utilizando dimensões lineares. Revista Ciência Agronômica, Fortaleza, v. 41, n. 4, p. 702-708, 2010. http://dx.doi.org/10.1590/s1806-66902010000400026

CANDIDO, W. S.; COELHO, M. F. B.; MAIA, S. S. S.; CUNHA, C. S. M.; SILVA, R. C. P. Modelo para estimar a área foliar de Combretum leprosum Mart. Acta Agronômica, Palmira, v. 62, n. 1, p. 37-41. 2013.

CARDOZO, N. P.; PARREIRA, M. C.; PANOSSO, A. R.; VOLPE, C. A. Modelagem da área foliar de duas cultivares de amendoim em função das dimensões lineares dos folíolos. Bioscience Journal, Uberlândia, v. 30, n. 1, p. 101-107. 2014.

CHO, Y. Y.; OH, S.; OH, M. M.; SON, J. E. Estimation of individual leaf area, fresh weight, and dry weight of hydroponically grown cucumbers (Cucumis sativus L.) using leaf length, width, and SPAD value. Scientia

Horticulturae, Amsterdam, v. 111, n. 4, p. 330-334. 2007. http://dx.doi.org/10.1016/j.scienta.2006.12.028

CRISTOFORI, V.; ROUPHAEL, Y.; GYVES, E. M.; BIGNAMI, C. A simple model for estimating leaf area of hazelnut from linear measurements. Scientia Horticulturae, Amsterdam, v. 113, n. 2, p. 221-225. 2007. http://dx.doi.org/10.1016/j.scienta.2007.02.006

DIAO, J.; LEI, X. D.; HONG, L. X.; RONG, J. T.; SHI, Q. Single leaf area estimation models based on leaf weight of eucalyptus in southern China. Journal of Forestry Research, v. 21, n. 1, p. 73-76. 2010. http://dx.doi.org/10.1007/s11676-010-0012-4

ELSNER, E. A.; JUBB JR, G. L. Leaf Area Estimation of 'Concord' Grape Leaves from Simple Linear Measurements. American Journal of Enology and Viticulture, Davis, v. 39, n. 1, p. 95-97. 1988.

FAVARIN, J. L.; DOURADO NETO, D.; GARCÍA, A. G.; VILLA NOVA, N. A.; FAVARIN, M. G. G. V. Equações para a Estimativa do Índice de Área Foliar do Cafeeiro. Pesquisa Agropecuária Brasileira, Brasília, v. 37, n. 6, p. 769-773. 2002. http://dx.doi.org/10.1590/s0100-204x2002000600005

FONSECA, C. E. L.; CONDÉ, R. C. C. Estimativa da área foliar em mudas de mangabeira (Harcornia speciosa Gom.). Pesquisa Agropecuária Brasileira, Brasília, v. 29, n. 4, p. 593-599. 1994.

GARDNER, F. P.; PEARCE, B. B.; MITCHELL, R. L. Physiology of crops plants. 2. Ed. Iowa, Biologia Platarum. 1990. 
GIUFFRIDA, F.; ROUPHAEL, Y.; TOSCANO, S.; SCUDERI, D.; ROMANO, D.; RIVERA, C. M.; COLLA, G.; LEONARDI, C. A simple model for nondestructive leaf area estimation in bedding plants.

Photosynthetica, Prague, v. 49, n. 3, p. 380-388. 2011. http://dx.doi.org/10.1007/s11099-011-0041-z

KUMAR, R.; SHARMA, S. Allometric model for nondestructive leaf area estimation in clary sage (Salvia sclarea L.). Photosynthetica, Prague, v. 48, n. 2, p. 313-316. 2010. http://dx.doi.org/10.1007/s11099-0100039-y

KVET, J.; MARSHALL, J. K. Assessment of leaf area and other assimilating plant surfaces. In SESTÁK, Z.; EATSKY, J.; JARVIS, P. G. Plant Photosynthetic Production: Manual of Methods, Netherlands. 1971, The Hague. p. 517-555.

LEITE, H. G.; ANDRADE, V. C. L. A method for conducting forest inventories without using volumetric equations. Revista Árvore, Viçosa, v. 26, n. 3, p. 321-328. 2002.

LIMA, R. T.; SOUZA, P. J. O. P.; RODRIGUES, J. C.; LIMA, M. J. A. Modelos para estimativa da área foliar da mangueira utilizando medidas lineares. Revista Brasileira de Fruticultura, Jaboticabal, v. 34, n. 4, p. 974-980. 2012. http://dx.doi.org/10.1590/s0100-29452012000400003

MA, L.; GARDNER, F. P.; SELAMAT, A. Estimation of leaf area and total mass measurements in peanut. Crop Science, Madison, v. 32, n. 2, p. 467-471. 1992.

http://dx.doi.org/10.2135/cropsci1992.0011183x003200020036x

MARACAJÁ, P. B.; MADALENA, J. A. S.; ARAÚJO, E.; LIMA, B. G.; LINHARES, P. C. F. Estimativa da área foliar de juazeiro por dimensões lineares do limbo foliar. Revista Verde de Agroecologia e

Desenvolvimento Sustentável, Pombal, v. 3, n. 4, p. 1-5. 2008.

MANIVEL, L.; WEAVER, R. J. Biometric correlations between leaf area and length measurements of 'Grenache' grape leaves. HortScience, Alexandria, v. 9, n. 1, p. 27-28. 1974.

MARSHALL, J. K. Methods for leaf area measurement of large and small leaf samples. Photosynthetica, Prague, v. 2, n. 1, p. 41-47. 1968.

MONTERO, F. J.; DE JUAN, J. A.; CUESTA, A.; BRASA, A. Non destructive methods to estimate leaf area in Vitis vinifera L. HortScience, Alexandria, v. 35, n. 4, p. 696-698. 2000.

NASCIMENTO, I. B.; FARIAS, C. H. A.; SILVA, M. C. C.; MEDEIROS, J. F.; SOBRINHO, J. E.; NEGREIROS, M. Z. Estimativa da área foliar do meloeiro. Horticultura Brasileira, Brasília, v. 20, n. 4, p. 555-558. 2002. http://dx.doi.org/10.1590/s0102-05362002000400009

PEKSEN, E. Non-destructive leaf area estimation model for faba bean (Vicia faba L.). Scientia Horticulturae, Amsterdam,v. 113, n. 4, p. 322-328. 2007. http://dx.doi.org/10.1016/j.scienta.2007.04.003

PIRES, R. C. M.; FOLEGATTI, M. V.; PASSOS, F. A. Estimativa da área foliar de morangueiro.

Horticultura Brasileira, Brasília, v. 17, p. 86-90. 1999. http://dx.doi.org/10.1590/s0102-05361999000200002

QUEIROZ, J. E.; SILVA, G. H.; NETO, A. G. S. Avaliação estatística da área foliar através de modelos de equações em duas espécies florestais. Revista Verde de Agroecologia e Desenvolvimento Sustentável, Pombal, v. 8, n. 1, p. 146-153. 2013.

SCHMILDT, E. R.; AMARAL, J. A. T.; SCHMILDT, O.; SANTOS, J. S. Análise comparativa de equações para estimativa da área foliar em Cafeeiros. Coffee Science, Lavras, v. 9, n. 2, p. 155-167. 2014. 
SEPÚLVEDA, G. R.; KLIEWER, W. M. Estimation of leaf area of two grapevine cultivars (Vitis vinifera L.) using laminae linear measurements and fresh weight. American Journal of Enology and Viticulture, Davis, v. 34, n. 4, p. 221-226. 1983.

SERDAR, U.; DEMIRSOY, H. Non-destructive leaf area estimation in chestnut. Scientia Horticulturae, Amsterdam, v. 108, n. 2; p. 227-230. 2006. http://dx.doi.org/10.1016/j.scienta.2006.01.025

SILVA, S. F.; PEREIRA, L. R.; CABANEZ, P. A.; AMARAL, J. A. T. Estimativas da área foliar de abieiros (Pouteria caimito) por meio de modelos alométricos. Agrarian Academy, Goiânia, v. 1; n. 1, p. 202-209. 2014. http://dx.doi.org/10.18677/agrarian_academy_2014_017

SILVA, R. T. L.; ANDRADE, A. C.; OLIVEIRA, L. M.; LIMA, L. G. S.; OLIVEIRA, R. L. L.; MELO, E. C.; OLIVEIRA NETO, C. F. Estimativa da área foliar de limoeiro-cravo usando dimensões lineares do limbo foliar. Nucleus, Ituverara, v. 10, n. 1, p. 91-98. 2013. http://dx.doi.org/10.3738/1982.2278.812

SILVA, A. C.; LEONEL, S.; SOUZA, A. P.; SOUZA, M. E.; TANAKA, A. A. Crescimento de figueira sob diferentes condições de cultivo. Pesquisa Agropecuária Tropical, Goiânia, v. 41, n. 4, p. 539-551, 2011. https://doi.org/10.5216/pat.v41i4.13223

SMART, R. E. Principles of grapevine canopy microclimate manipulation with implications for Yield and Quality: A Review. American Journal of Enology and Viticulture, Davis, v. 36, n. 3, p. 230-239. 1985.

SMITH, R. J.; KLIEWER, W. M. Estimation of Thompson Seedless Grapevine leaf area. American Journal of Enology and Viticulture, Davis, v. 35, n. 1, p. 16-22. 1984.

SOUZA, A. P.; SILVA, A. C.; LEONEL, S.; SOUZA, M. E.; TANAKA, A. A. Estimativas da área da folha de Figueiras 'Roxo De Valinhos' usando dimensões lineares do limbo foliar. Ciência Rural, Santa Maria, v. 44, n. 7, p. 1172-1179. 2014. http://dx.doi.org/10.1590/0103-8478cr20130699

SOUZA, A. P.; MOTA, L. L.; ZAMADEI, T.; MARTIM, C. C.; ALMEIDA, F. T.; PAULINO, J. Classificação climática e balanço hídrico climatológica no estado de Mato Grosso. Nativa, Sinop, v. 1, n. 1, p. 34-43, 2013. http://dx.doi.org/10.14583/2318-7670.v01n01a07

STRIK, B. C.; PROCTOR, J. T. A. Estimating the area of trifoliate and unequally imparipinnate leaves of strawberry. HortScience, Alexandria, v. 20, n. 6, p. 1072-1074. 1985.

TOEBE, M; FILHO, A. C.; LOOSE, L. H.; HELDWEIN, A. B.; ZANON, A. J. Área foliar de feijão-vagem (Phaseolus vulgaris L.) em função de dimensões foliares. Semina: Ciências Agrárias, Londrina, v. 33, n. 6-1, p. 2491-2500. 2012. http://dx.doi.org/10.5433/1679-0359.2012v33supl1p2491

THIERSCH, A. Eficiência das distribuições diamétricas para prognose da produção de Eucalyptus camaldulensis. 155p. Dissertação (Mestrado em Engenharia Florestal) - Universidade Federal de Lavras, Lavras, 1997.

TSIALTAS, J. T.; KOUNDOURAS, S.; ZIOZIOU, E. Leaf area estimation by simple measurements and evaluation of leaf area prediction models in cabernet-sauvignon grapevine leaves. Photosynthetica, Prague, v. 46, n. 3, p. 452-456. 2008. http://dx.doi.org/10.1007/s11099-008-0077-x

VIDAL, W. N.; VIDAL, M. R. R. Botânica Organografia: quadros sinóticos ilustrados de fanerógamos. Ed.4, Viçosa: UFV, 2003. 124 p.

WILLIAMS, L. E. Growth of 'Thompson Seedless' grapevines. I. Leaf area development and dry weight distribution. Journal of the American Society for Horticultural Science, Alexandria, v. 11, n. 2, p. 325-330. 1987.

WILLMOTT, C. J. On the validation of models. Physical Geography, Norwich, v. 2, n. 2, p. 184-194. 1981. 